\title{
miR-762 Promotes Malignant Development of Head and Neck Squamous Cell Carcinoma by Targeting PHLPP2 and FOXO4
}

This article was published in the following Dove Press journal: OncoTargets and Therapy

\author{
Shuai Chen ${ }^{1-3}$ \\ Jian-Yun Zhang $\mathbb{( D}^{1,3}$ \\ Li-Sha Sun $\mathbb{D}^{2,3}$ \\ Xue-Fen $\mathrm{Li}$ (D) $^{2,3}$ \\ Jia-Ying Bai \\ He-Yu Zhang ${ }^{2,3}$ \\ Tie-Jun $\mathrm{Li}^{1,3}$
}

'Department of Oral Pathology, Peking University School and Hospital of Stomatology, Beijing 10008I, People's Republic of China; ${ }^{2}$ Central Laboratory, Peking University School and Hospital of Stomatology, Beijing 10008I, People's Republic of China; ${ }^{3}$ Research Unit of Precision Pathologic Diagnosis in Tumors of the Oral and Maxillofacial Regions, Chinese Academy of Medical Sciences, Beijing 10008I, People's Republic of China
Correspondence: Tie-Jun Li Department of Oral Pathology, Peking University School and Hospital of Stomatology, Beijing 10008I, People's Republic of China

$\mathrm{Tel} / \mathrm{Fax}+8601082195203$

Email litiejun22@vip.sina.com

He-Yu Zhang

Central Laboratory, Peking University School and Hospital of Stomatology,

Beijing 10008I, People's Republic of China

$\mathrm{Tel} / \mathrm{Fax}+8601082195770$

Email zhangheyu1983@sina.cn
Background: Head and neck squamous cell carcinoma (HNSCC) is among the most common malignant tumors worldwide. This study, investigated the role of microRNA (miR)-762 in regulating HNSCC progression.

Materials and methods: The expression levels of miR-762 in HNSCC tissues were measured by quantitative reverse transcription polymerase chain reaction (qRT-PCR). Statistical analyses were performed to investigate the association of miR-762 with clinicopathological features in patients with HNSCC. Cell proliferation and migration were examined by cell counting (CCK-8) and IncuCyte assays. Target genes of miR-762 were screened using bioinformatics tools and microarrays, and confirmed using a luciferase activity reporter assay, qRT-PCR and Western blot analysis. Recuse experiments were performed to detect whether target genes mediated the effects of miR-762 on HNSCC cells. The in vivo effects of miR-762 were verified using tumor xenografts.

Results: HNSCC clinical specimens showed high expression levels of miR-762, which positively correlated with tumor-node-metastasis (TNM) stage and poor prognosis of HNSCC. miR-762 overexpression promoted the proliferation and migration of HNSCC cells in vitro. In addition, overexpression of miR-762 upregulated the expression of phosphorylated AKT (p-AKT) and mesenchymal markers (N-cadherin and vimentin), but suppressed epithelial marker (E-cadherin) expression. miR-762 also promoted HNSCC tumor growth in vivo. PH domain and leucine-rich repeat protein phosphatase 2 (PHLPP2) and Forkhead box O4 (FOXO4) were direct target genes of miR-762. HNSCC tissues had low expression levels of PHLPP2 and FOXO4, showing a negative correlation with miR-762 expression. Moreover, silencing of PHLPP2 and FOXO4 mimicked the tumor-promotive effects of miR-762 on HNSCC cells. Notably, overexpression of PHLPP2 and FOXO4 abolished the pro-tumoral function of miR-762 on cell proliferation and migration.

Conclusion: miR-762 promotes HNSCC progression by targeting PHLPP2 and FOXO4. Therefore, miR-762 might be a potential diagnostic or therapeutic target for HNSCC.

Keywords: miR-762, FOXO4, PHLPP2, proliferation, migration, HNSCC

\section{Introduction}

Head and neck cancer accounts for $4.8 \%$ of global cancers. ${ }^{1}$ In the United States, there will be approximately 117,480 new cases of head and neck cancer and 16,790 deaths in $2019 .^{2}$ Approximately 135,000 new cases occurred in China in 2015, and about 70, 000 people died of the disease. ${ }^{3}$ Head and neck squamous cell carcinoma (HNSCC) accounts for $90 \%$ of head and neck cancer and is the sixth 
most common type of malignant tumor worldwide. ${ }^{4}$ According to GLOBOCAN 2012, the age-standardized incidence of HNSCC in China is estimated to be 2.7 per 100,000 people. ${ }^{5}$ Despite advances in diagnosis and treatment, the 5-year survival rate of patients with HNSCC remains poor due to its high propensity for local and distant metastasis. ${ }^{6}$ The initiation and progression of HNSCC are linked to multiple risk factors including HPV, diet, detrimental habits, genetic background and geographic factors; ${ }^{7}$ however, the underlying molecular mechanisms are unclear. Therefore, understanding the mechanism of HNSCC progression is important to identify therapies for improving the clinical outcomes of HNSCC.

MicroRNAs (miRNAs), a class of non-coding singlestranded RNA molecules of 18-25 nucleotides in length, play a post-transcriptional regulatory role via complementary pairing with the $3^{\prime}$ untranslated region (UTR) of target mRNAs. ${ }^{8}$ miRNAs participate in a number of biological events, such as drug sensitivity, infection, stem cell differentiation, pluripotency maintenance and immune responses. ${ }^{8-11}$ Recently, a number of miRNAs have been reported to be dysregulated in various cancer types, including HNSCC. ${ }^{12,13}$ For instance, miR-141 shows decreased expression in HNSCC tissues and exerts tumor-suppressive roles by inhibiting the expression of epidermal growth factor receptor (EGFR). ${ }^{14}$ miR-200 facilitates the epithelial-mesenchymal transition (EMT) in HNSCC cells and promotes HNSCC progression. ${ }^{15}$ miR-762 functions as a tumor promoter, as high expression of miR-762 promotes breast cancer cell proliferation and invasion by targeting Interferon regulatory factor 7 (IRF7) expression. ${ }^{16}$ Furthermore, miR-762 upregulates the Wnt signaling pathway to promote the development of ovarian cancer. ${ }^{17}$ However, the biological function of miR-762 in HNSCC remains unclear.

This study examined miR-762 expression in HNSCC and its association with clinicopathological features in patients with HNSCC. In vitro and in vivo functional assays were performed to explore the detailed effects of miR-762 on HNSCC. We revealed that HNSCC patients with high expression of miR-762 showed poorer overall survival compared to those with low miR-762 expression. Furthermore, miR-762 promoted the progression of HNSCC, and both PHLPP2 and FOXO4 were novel target genes of miR-762 in HNSCC.

\section{Materials and Methods}

\section{Clinical Specimens and Cell Culture}

Pairs of HNSCC tissue and normal adjacent tissue (NAT) samples were collected from 68 patients at the Peking University Hospital of Stomatology from 2013 to 2016. We excluded surgical specimens from HNSCC patients who underwent preoperative radiotherapy and chemotherapy. NAT samples were dissected $2 \mathrm{~cm}$ from the tumor edge. All tissues were immediately preserved in liquid nitrogen and stored at $-80^{\circ} \mathrm{C}$. Frozen tissues were stained with hematoxylin and eosin and the pathology was subsequently confirmed by two experienced pathologists. Signed informed consent was obtained from the patients in accordance with the Declaration of Helsinki, and the Ethics Committee of the Peking University Hospital of Stomatology approved this study.

\section{Cell Culture}

Human HNSCC cell lines (CAL27, FaDu, SCC-9, SCC-15 and SCC-25) were purchased from the American Type Culture Collection (Manassas, VA, USA) and the human immortalized epithelial cell line $\mathrm{HaCaT}$ was purchased from the Cell Lines Service (Eppelheim, Germany). SCC9, SCC-15, and SCC-25 cells were cultured in Dulbecco's modified Eagle medium (DMEM)/F12 (Gibco, Thermo Fisher Scientific, Inc., Waltham, MA, USA) supplemented with hydrocortisone $(400 \mathrm{ng} / \mathrm{mL})$ and $10 \%$ fetal bovine serum (FBS). CAL27, FaDu, and $\mathrm{HaCaT}$ cells were cultured in DMEM (Gibco) supplemented with 10\% FBS. Cells were grown in a $5 \% \mathrm{CO}_{2}$ humidified incubator at $37^{\circ} \mathrm{C}$.

\section{Cell Transfection and Infection}

miR-762 mimic (mimic), miR-762 inhibitor (inhibitor), and negative control (miR-NC) were purchased from Guangzhou RiboBio Co., Ltd. (Guangzhou, Guangdong, China). The pcDNA3.1-FOXO4 (pc-FOXO4) and pcDNA3.1-PHLPP2 (pc-PHLPP2) plasmids were synthesized by Shanghai Generay Co., Ltd. (Shanghai, China). Small interfering RNAs (siRNAs) targeting FOXO4 (siFOXO4) and PHLPP2 (siPHLPP2) and the negative control siRNA (siNC) were synthesized by Suzhou GenePharma Co., Ltd. (Suzhou, Jiangsu, China). Lentiviruses for miR-762 and Mock were synthesized by Suzhou GenePharma Co., Ltd. All transfection procedures were performed using Lipofectamine ${ }^{\mathrm{TM}} 3000$ (Invitrogen, Carlsbad, CA, USA) according to the manufacturer's instructions. 


\section{Microarrays}

The human gene expression profile was detected using Affymetrix's PrimeView ${ }^{\mathrm{TM}}$ Human Gene Expression Array, and performed by Beijing CapitalBio Corporation (Beijing, China) according to the manufacturer's instructions. ${ }^{18}$ All primary data from the microarray analyses are available by accessing the Gene Expression Omnibus (GEO accession: GSE122328).

\section{RNA Extraction and Quantitative Real-Time Polymerase Chain Reaction (qRT-PCR) Analyses}

Total RNA was isolated using TRIzol reagent (Invitrogen) according to the manufacturer's instructions. The primers for miR-762 and U6 were purchased from Guangzhou RiboBio Co. Total RNA $(1 \mu \mathrm{g})$ was reverse transcribed into cDNA using the miDETECT A Track ${ }^{\mathrm{TM}}$ miRNA qRT-PCR Starter Kit (Guangzhou RiboBio Co.) to generate the miRNA, and PrimeScript RT Reagent Kit (Takara Biotechnology Co., Ltd., Dalian, China) was used to generate FOXO4 and PHLPP2. The FastStart universal SYBR-Green Master kit (Roche, Mannheim, Germany) was used to detect the mRNA expression of miR-762, FOXO4, and PHLPP2. Relative mRNA expression was calculated using the $2^{-\Delta \Delta \mathrm{Ct}}$ method after normalizing to U6 or GAPDH levels. The primer sequences are listed in Table 1.

\section{Cell Counting (CCK-8) Assay}

HNSCC cells were seeded in 96-well plates (4000 cells/ well) and transfected after $24 \mathrm{~h}$. CCK-8 solution $(10 \mu \mathrm{L} /$ well) was added to each well at the indicated time, and the samples were incubated for $2 \mathrm{~h}$ at $37^{\circ} \mathrm{C}$. Then, the absorbance was detected at $450 \mathrm{~nm}$ using a microplate reader (Bio-Rad Laboratories, Hercules, CA, USA).

Table I Primers Used in This Study

\begin{tabular}{|l|l|}
\hline Name & Sequence \\
\hline GAPDH forward & 5'-ACAGTCAGCCGCAT-3' \\
GAPDH reverse & 5'-GACAAGCTTCCCGT-3' \\
FOXO4 forward & 5'-CTTTCTGAAGACTGGCAGGAATGTG-3' \\
FOXO4 reverse & 5'-GATCTAGGTCTATGATCGCGGCAG-3' \\
PHLPP2 forward & 5'-ATGGAGCAGACACTACCACTG-3' \\
PHLPP2 reverse & 5'-GCAAAGGACGAGATGTAAGTCA-3' \\
U6 forward & 5'-CTCGCTTCGGCAGCACA-3' \\
U6 reverse & 5'-AACGCTTCACGAATTTGCGT-3' \\
\hline
\end{tabular}

IncuCyte Proliferation and Migration Assay HNSCC cells were seeded in 96-well plates for the proliferation (4000 cells/well) and migration (20,000 cells/well) assays. The proliferation assays were monitored using the IncuCyte ZOOM Live-Cell Imaging system (IncuCyte system, Essen Bioscience, Ann Arbor, MI, USA). The adhered cells in the migration assay plates were starved in serum-free medium for $12 \mathrm{~h}$, and a wound was scratched in one well using the 96-pin wound-marker according to the manufacturer's instructions (Essen Bioscience). The plates were monitored and photographed every 12 or $6 \mathrm{~h}$ for $96 \mathrm{~h}$, and the percentages of cell confluence or wound widths were calculated over time. All experiments were performed at least three times in triplicate.

\section{Xenograft Assay}

This study was carried out in strict accordance with the national standard: laboratory animal-guide for ethnic al review of animal welfare (GB/T 35892-2018), and approved by the Peking University Biomedical Ethics Committee. Logarithmically growing CAL27 cells transfected with Lenti-miR-762 or Lenti-Mock were prepared as cell suspensions $\left(3.0 \times 10^{6}\right.$ cells $\left./ 100 \mu \mathrm{L}\right)$ and subcutaneously injected into the left anterior axilla of 5-week-old BALB/c female nude mice ( $\mathrm{N}=5$, per group). Tumor width and length were measured every 4 days with a caliper, and the volume of tumor xenografts was calculated using the following formula: volume $=1 / 2\left(\right.$ length $\times$ width $\left.^{2}\right)$. Four weeks after inoculation, mice were sacrificed by cervical dislocation, and tumors were collected for measurement and analyses.

\section{Luciferase Reporter Assay}

The wild-type and mutant $3^{\prime}$ UTRs of FOXO4 and PHLPP2 containing the predicted miR-762 binding sites were cloned into psiCHECK-2 vectors to generate miRFOXO4-wt/mut3'UTR and miR-PHLPP2-wt/mut3'UTR. The constructed plasmids and miR-762 mimic or miRNC were co-transfected into SCC-9 and CAL27 cells in 12-well plates. After $24 \mathrm{~h}$, the transfected cells were harvested, and the Dual-Luciferase Assay Kit (Promega Corporation, Madison, WI, USA) was used according to the manufacturer's instructions.

\section{Western Blot}

Cells were lysed, and protein extracts were separated using $10 \%$ sodium dodecyl sulfate polyacrylamide gel electrophoresis and transferred to polyvinylidene difluoride 
membranes (Millipore, Billerica, MA, USA). After blocking the membranes, rabbit polyclonal antibodies against FOXO4 (1:1000, ab128908, Abcam, Cambridge, UK) and PHLPP2 (1:1000, ab71973, Abcam), and a GAPDH monoclonal antibody (1:4000, \#2118, Cell Signaling Technology, Danvers, MA, USA) were incubated with the membranes. The anti-rabbit or anti-mouse horseradish peroxidase-conjugated secondary antibodies (1:5000, \#7074, Cell Signaling Technology) were then added to the blots and the blots were exposed using an Image Reader LAS-4000 (Fujifilm, Japan).

\section{Immunohistochemistry (IHC)}

Tumor sections fixed in $4 \%$ formaldehyde were embedded in paraffin, dewaxed, and rehydrated. Antigen retrieval was performed by boiling in $10 \mathrm{mM}$ sodium citrate buffer (pH 6.0) for $30 \mathrm{~min}$. Then, the samples were treated with $3 \%$ hydrogen peroxide for $10 \mathrm{~min}$ and blocked with $5 \%$ goat serum for $30 \mathrm{~min}$ at room temperature. The sections were incubated with anti-FOXO4 (1:100, 21535-1-AP; Proteintech, Chicago, IL, USA), anti-PHLPP2 (1:100, 25244-1-AP; Proteintech), or normal rabbit IgG (\#2729; Cell Signaling Technology) as a control at $4^{\circ} \mathrm{C}$ overnight. After washing, the sections were stained using the Catalyzed Signed Amplification System Kit (Dako, Carpinteria, CA, USA; code k5007).

\section{Kaplan-Meier Survival Analysis}

The Kaplan-Meier plotter (www.kmplot.com) was used to assess the effects of miR-762 expression on the overall survival of patients with HNSCC. The cut-off value was auto-selected in the algorithm.

\section{Statistical Analyses}

Statistical analyses were performed using SPSS version 17.0. The association between miR-762 expression and the clinical features of patients with HNSCC was determined using the Chi-squared test. Spearman correlation analysis was used to examine the expression correlation between miR-762 and FOXO4/PHLPP2 mRNA in HNSCC samples. The mean comparison between groups was performed using the two-tailed Student's $t$-test. Overall survival rates were calculated by the KaplanMeier method and analyzed using a log rank test. Data were presented as the mean \pm standard deviation. $P$ values $<0.05$ were considered to indicate statistical significance.

\section{Results Upregulation of miR-762 Is Associated with Poor Prognosis in HNSCC}

To detect the expression of miR-762 in HNSCC tissues, we examined miR-762 expression in 68 pairs of HNSCC and NAT samples using qRT-PCR. The results showed upregulated expression of miR-762 in HNSCC samples compared to NAT samples (Figure 1A). In addition, we tested the expression of miR-762 in five HNSCC cell lines (FaDu, SCC-25, SCC-15, SCC-9 and CAL27), with the human immortalized epithelial cell line (HaCaT) serving as control. miR-762 expression was higher in the five HNSCC cell lines than in HaCaT cells (Figure 1B).

To assess the correlation between miR-762 expression and the clinical characteristics of patients with HNSCC, we divided the HNSCC cases into two groups according to the median value of miR-762 expression in HNSCC tissues. The results showed a high correlation of miR-762 expression with TNM stage in patients with HNSCC. However, no significant
A

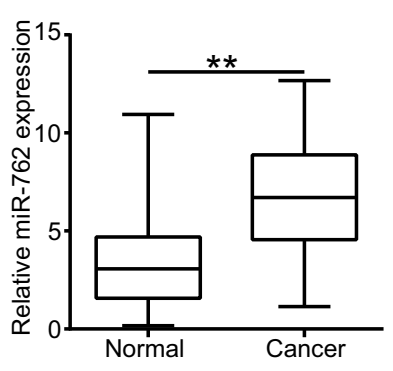

B

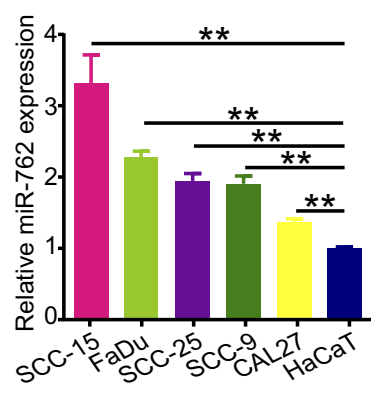

C

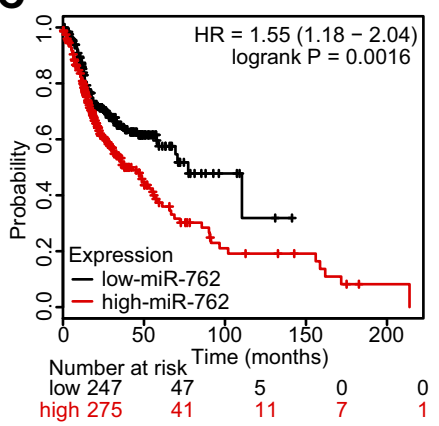

D

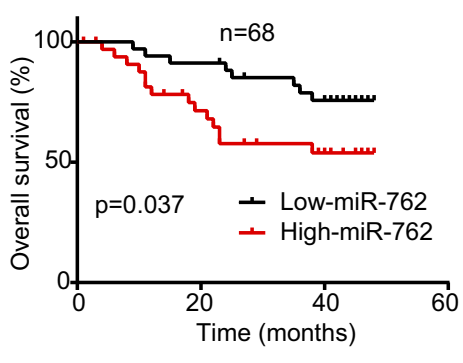

Figure I miR-762 was high expression in HNSCC tissues and cell lines.

Notes: (A) Relative expression of miR-762 was evaluated by RT-qPCR in 68 pairs of HNSCC and NAT tissues; (B) miR-762 expression in HaCaT and five HNSCC cell lines was evaluated using RT-qPCR. (C) The result of Kaplan-Meier plotter website showing the survival curve between HNSCC patients with high- and low-miR-762 expression. (D) The overall survival rates (OS) of $68 \mathrm{HNSCC}$ cases from Peking University Hospital of Stomatology were calculated dependent on miR-762 expression. $* * p<0.01$. 
relationship was detected between miR-762 expression and age, sex, and smoking status (Table 2). The Kaplan-Meier survival curve (analyzed using the KM Plotter website) indicated that patients with high miR-762 expression tended to have poorer overall survival compared to those with high miR-762 expression (Figure 1C). Our data consistently showed that patients with high miR-762 expression had shorter overall survival than those with low miR-762 expression (Figure 1D). These data suggest that upregulated miR762 is closely related to the progression of HNSCC and might be a potential predictive biomarker of HNSCC outcome.

\section{miR-762 Promotes Proliferation and Migration of HNSCC Cells in vitro}

Considering that HNSCC showed upregulated miR-762 expression, we speculated that miR-762 might play a role in promoting HNSCC progression. To verify this hypothesis, gain and loss-of-function assays were performed using transient transfection strategies. We transfected

Table 2 Correlation Between the Clinicopathologic Characteristics and Expression of miR-762 in HNSCC Cases

\begin{tabular}{|c|c|c|c|c|}
\hline \multirow[t]{2}{*}{ Characteristics } & \multirow[t]{2}{*}{ Number } & \multicolumn{2}{|c|}{$\begin{array}{l}\text { Patients with miR-762 } \\
\text { Expression }\end{array}$} & \multirow[t]{2}{*}{$p$-value } \\
\hline & & Low & High & \\
\hline \multicolumn{5}{|l|}{ Age (years) } \\
\hline$\geq 60$ & 25 & $9(36 \%)$ & $16(64 \%)$ & 0.05 \\
\hline$<60$ & 43 & 25 (58.14\%) & $18(41.86 \%)$ & \\
\hline \multicolumn{5}{|l|}{ Gender } \\
\hline Male & 48 & 23 (47.92\%) & 25 (52.08\%) & 0.76 \\
\hline Female & 20 & $11(55 \%)$ & $9(45 \%)$ & \\
\hline \multicolumn{5}{|l|}{ Smoking status } \\
\hline Smokers & 35 & $18(51.43 \%)$ & 17 (48.57\%) & 0.71 \\
\hline Nonsmokers & 33 & $16(48.48 \%)$ & 17 (5।.52\%) & \\
\hline \multicolumn{5}{|l|}{ TNM stage } \\
\hline I-II & 33 & $20(60.61 \%)$ & 13 (39.39\%) & $<0.01$ \\
\hline III-IV & 35 & $14(40 \%)$ & $21(60 \%)$ & \\
\hline \multicolumn{5}{|l|}{ T classification } \\
\hline T1-T2 & 46 & 25 (54.35\%) & 21 (45.65\%) & 0.14 \\
\hline T3-T4 & 22 & 9 (40.91\%) & 13 (59.09\%) & \\
\hline \multicolumn{5}{|l|}{$\mathrm{N}$ classification } \\
\hline No-NI & 48 & $26(54.17 \%)$ & 22 (45.83\%) & 0.56 \\
\hline N2-N3 & 20 & $9(45 \%)$ & II (55\%) & \\
\hline \multicolumn{5}{|l|}{ Distant metastasis } \\
\hline Yes & 5 & $2(40 \%)$ & $3(60 \%)$ & 0.35 \\
\hline No & 63 & $32(50 \%)$ & $31(49.21 \%)$ & \\
\hline
\end{tabular}

Note: Distant metastasis represented the $M$ classification.
SCC-9 and CAL27 cells (low levels of miR-762 expression) with miR-762 mimic or miR-NC, and transfected SCC-15 cells (high expression levels of miR-762) with miR-762 inhibitor or miR-NC. qRT-PCR analyses showed that miR-762 mimic or miR-762 inhibitor significantly upregulated or downregulated miR-762 expression, respectively (Figure $2 \mathrm{~A}$ and $\mathrm{B}$ ).

The proliferation ability of HNSCC cells was investigated using CCK-8 and IncuCyte proliferation assays. The results indicated that miR-762 mimic significantly promoted the proliferation of CAL27 and SCC-9 cells, whereas the knockdown of miR-762 significantly inhibited proliferation of SCC-15 cells (Figure 2C and D). In addition, the IncuCyte migration assay results demonstrated that miR-762overexpressing SCC-9 and CAL27 cells had stronger migratory ability compared to the miR-NC-transfected group, whereas SCC-15 cells transfected with miR-762 inhibitor had weaker migratory ability (Figure 2E). These results indicated that miR-762 overexpression promotes the malignant progression of HNSCC cells in vitro.

Western blot analyses revealed that miR-762 mimic enhanced the expression of $\mathrm{p}$-AKT, N-cadherin, and vimentin, but suppressed E-cadherin expression in SCC-9 and CAL27 cells (Figure 2F). Concordantly, opposite results were detected with miR-762 inhibitor in SCC-15 cells (Figure 2F).

\section{PHLPP2 and FOXO4 are Direct Targets of miR-762}

To further identify target mRNAs of miR-762, we upregulated miR-762 expression by transfecting miR-762 mimic into SCC-9 cells and screened the changes in mRNA level by microarray (Figure $3 \mathrm{~A}$ and $\mathrm{B}$ ). In addition, the target mRNAs were predicted by TargetScan7.0 (http://www.tar getscan.org/). These two approaches identified a total of 151 candidate genes (Figure 3C). Based on the oncogenic effects of miR-762 in HNSCC, we focused on the tumor suppressor genes PHLPP2 and FOXO4 as putative targets for further investigation. In addition, pathway enrichment analyses indicated that miR-762 was closely related to the PI3K pathway, which was consistent with our Western blot results (Figure 3D).

Dual-luciferase reporter assay results showed that overexpressed miR-762 significantly inhibited luciferase activity from PHLPP2-wt or FOXO4-wt promoters, whereas cells harboring PHLPP2-mut or FOXO4-mut promoters were not susceptible to the effects of miR-762 expression. These 
A

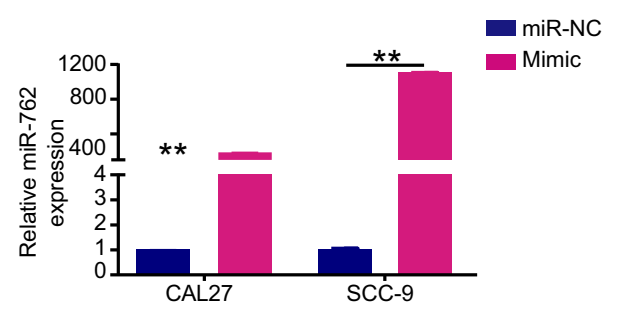

C

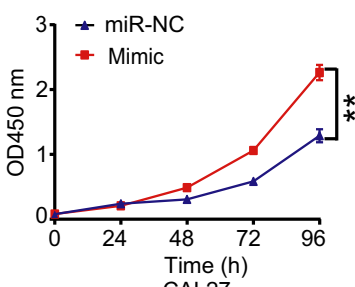

D

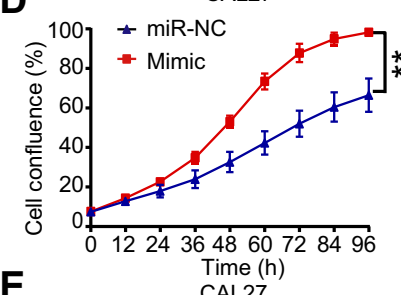

E

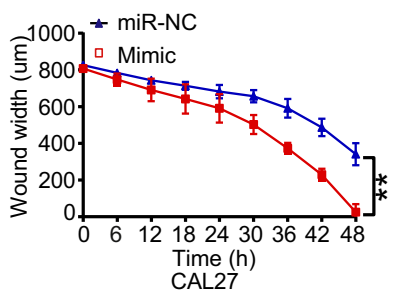

CAL27

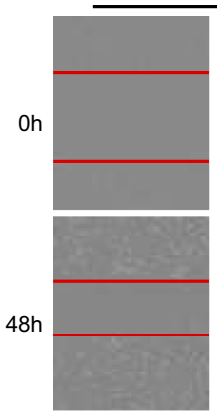

miR-NC

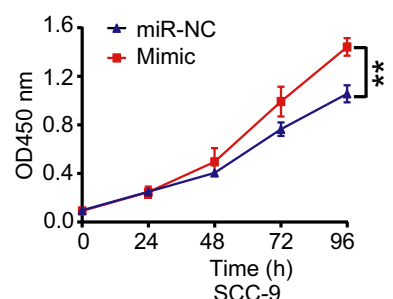

SCC-9

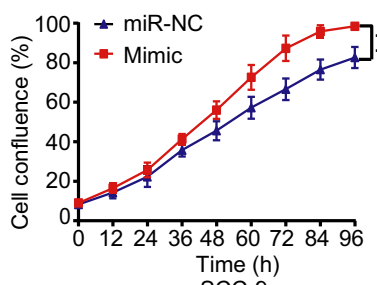

SCC-9

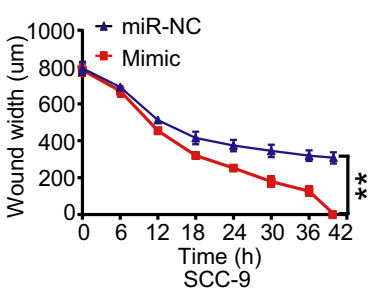

SCC-9

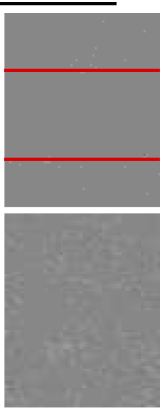

Mimic

Oh
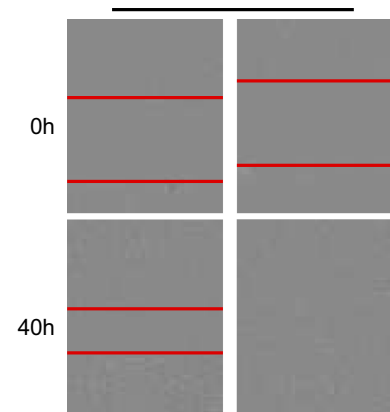

miR-NC

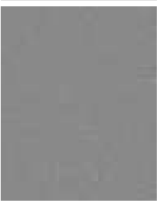

Mimic
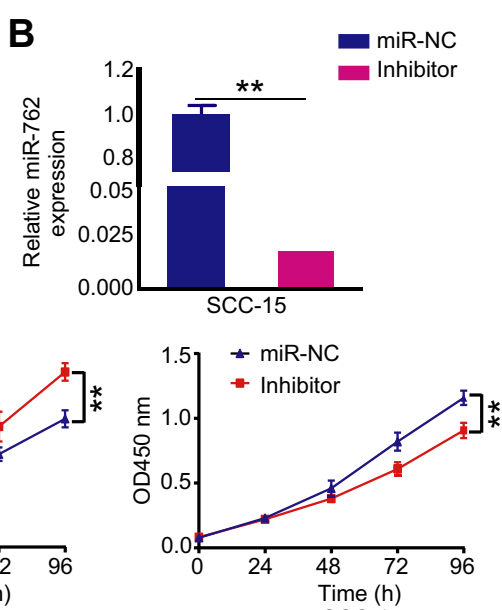

SCC-15
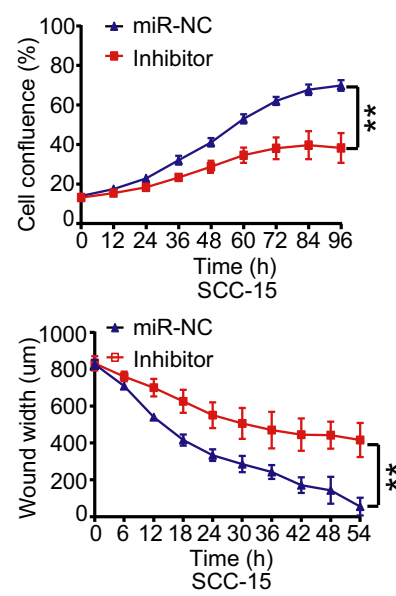

SCC-15
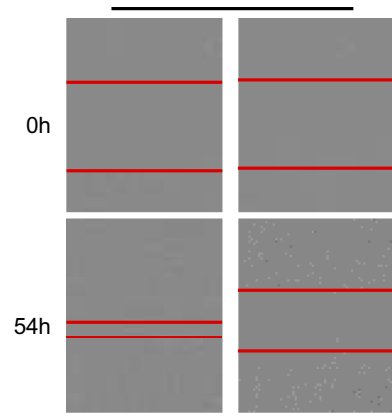

miR-NC

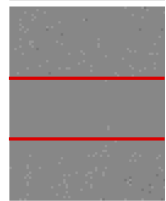

Inhibitor

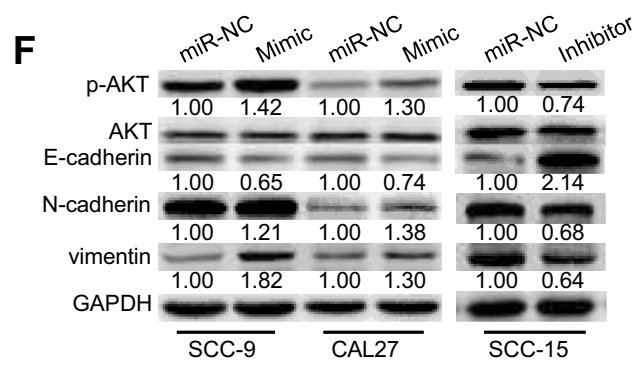

Figure 2 Enhanced expression of miR-762 promoted HNSCC cell proliferation and migration in vitro.

Notes: (A and B) CAL27 and SCC-9 cells were transfected with miR-762 mimics and miR-NC, SCC-I5 cells were transfected with miR-762 inhibitor and miR-NC. RTqPCR was used to detect the expression of miR-762 in SCC-9, CAL27 and SCC-15 cells treated as above described. (C-D) CCK-8 and IncuCyte proliferation assays were used to detect the proliferation of CAL27, SCC-9 and SCC-I5 cells treated as above described. (E) IncuCyte wound-healing assays were used to detect the migration of CAL27, SCC-9 and SCC-15 cells and as evidenced in the following light microscopy images. (F) Western blot analysis of p-AKT, E-cadherin, N-cadherin and vimentin expression in SCC-9, CAL27 and SCC-15 cells treated as above described. **p $<0.01$. 
A

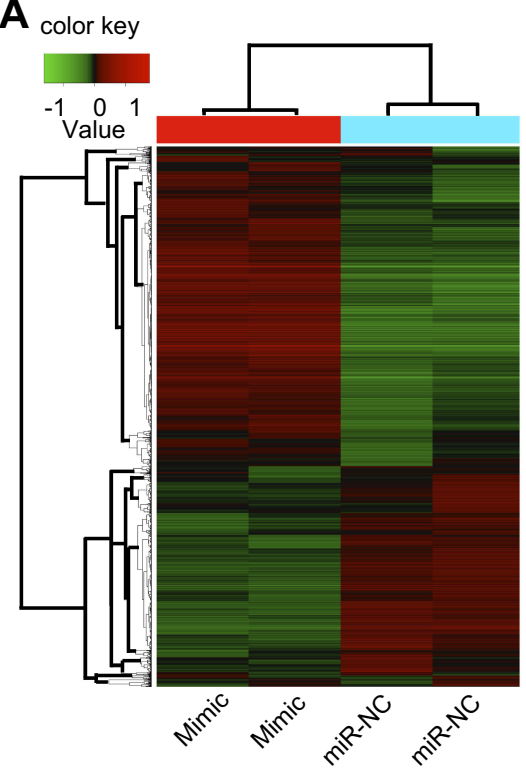

B

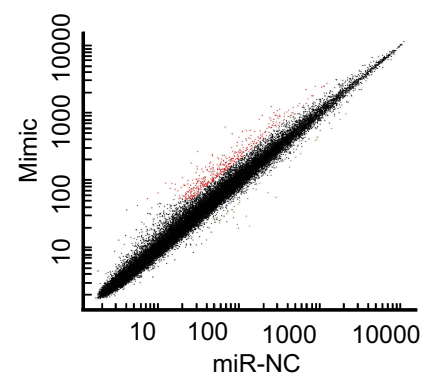

C

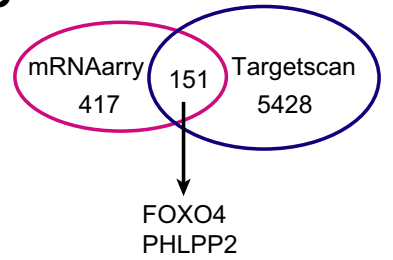

D

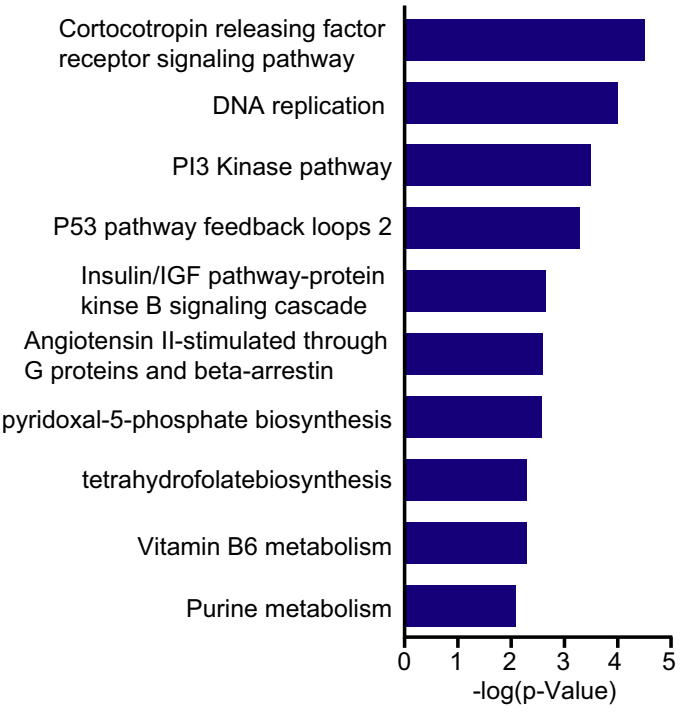

E
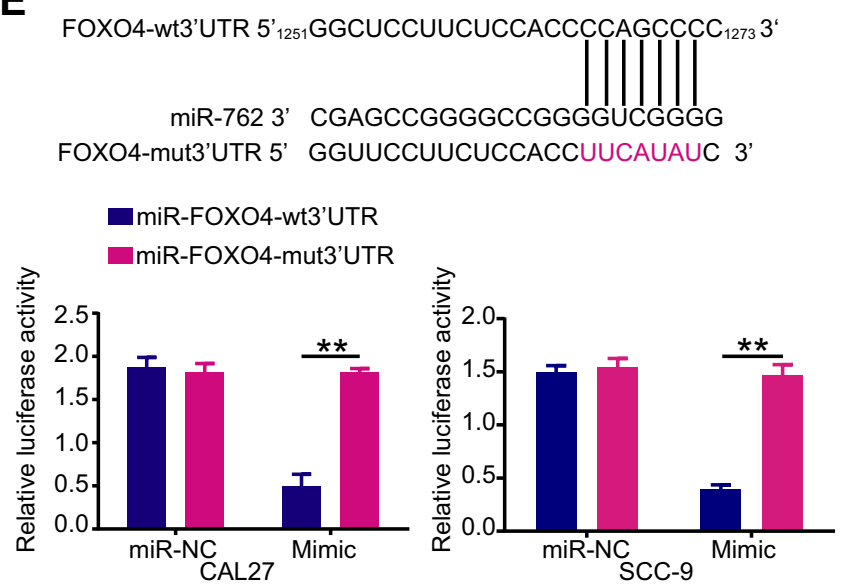

F

PHLPP2-wt3'UTR 5' ${ }_{382}$ CCUCAGCCACCAUGCCCAGCCCU $_{404} 3$ '

miR-762 3' CGAGCCGGGGCCGGGGUCGGGG PHLPP2-mut3'UTR 5' CCUCAGCCACCAUGCUACUACUU 3'

Figure 3 Screening of miR-762 target gene.

Notes: (A) A heatmap represented unsupervised hierarchical clustering of mRNA expression level in SCC-9 cells transfected with miR-762 mimics or miR-NC. (B) Scatter plot presentation of genes with a cutoff of 2/0.5. (C) Venn diagram of potential targets of miR-762 predicted by TargetScan and screened by microarrays. (D) The top ten signaling pathways through pathway enrichment were listed by PANTHER. (E and F) Predicted miR-762 target sequences in mRNA 3'UTR of FOXO4 and PHLPP2 and the positions of mutated nucleotides (red). Luciferase reporter assays of cells co-transfected with plasmids containing PHLPP2-wt/mut3'UTR or FOXO4-wt/mut3'UTR and miR762 mimics or miR-NC sequences. $* * p<0.01$.

results indicated that miR-762 could directly bind to the mRNA 3' UTRs of PHLPP2 and FOXO4 (Figure 3E and F).

qRT-PCR and Western blot analyses further verified the relationship between miR-762 and PHLPP2/FOXO4 at the mRNA and protein levels, respectively. Ectopic expression of miR-762 dramatically decreased the expression of PHLPP2 and FOXO4 in CAL27 and SCC-9 cells (Figure 4A, B, and D). By contrast, downregulating miR-762 expression with miR-762 inhibitor increased PHLPP2 and FOXO4 expression in SCC-15 cells (Figure 4C and D). These results demonstrated that PHLPP2 and FOXO4 are direct targets of miR-762.
qRT-PCR revealed low expression of PHLPP2 and FOXO4 in HNSCC tissues compared to that in NAT samples (Figure 4E). Moreover, Pearson correlation analyses showed a significant inverse correlation between the expression of PHLPP2/FOXO4 and miR-762 in HNSCC tissues (Figure 4F).

\section{PHLPP2 and FOXO4 Mediate the Role of miR-762 in HNSCC Cells}

Given that miR-762 suppressed PHLPP2/FOXO4 expression and promoted HNSCC progression, rescue assays were conducted to investigate whether PHLPP2 and FOXO4 

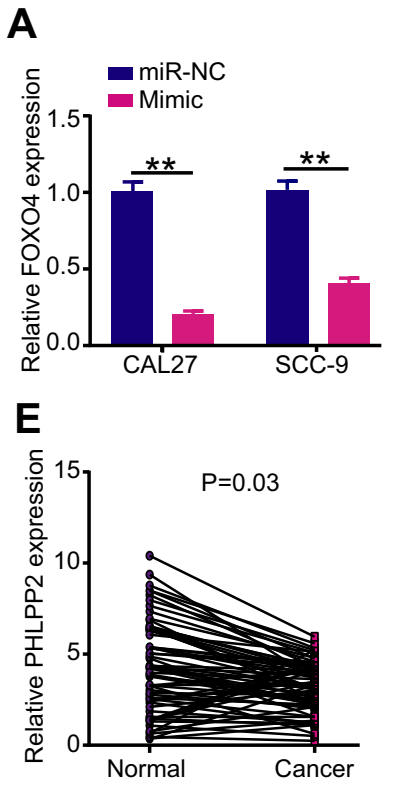
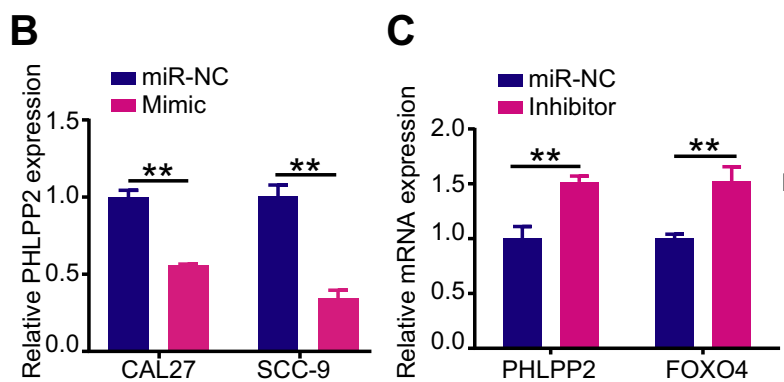

D

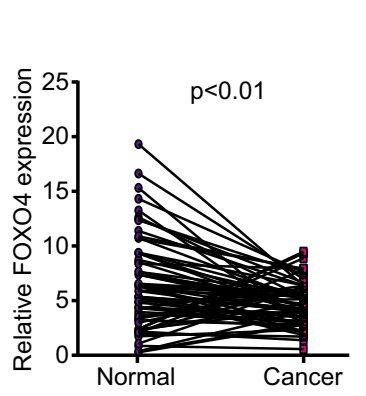

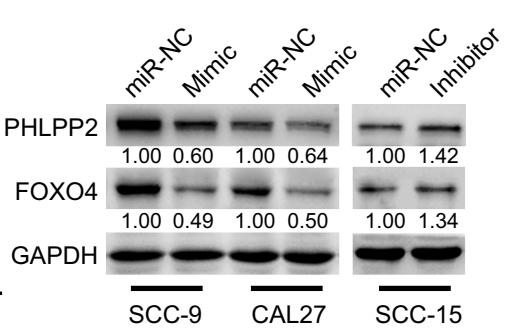

$\mathbf{F}$

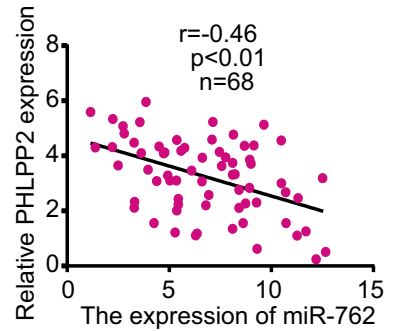

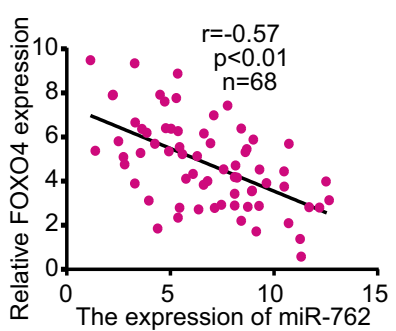

Figure 4 PHLPP2 and FOXO4 were direct targets of miR-762.

Notes: (A-D) CAL27 and SCC-9 cells were transfected with miR-762 mimics and miR-NC, SCC-15 cells were transfected with miR-762 inhibitor and miR-NC. RT-qPCR and Western blot were used to examine the mRNA and protein expression levels of PHLPP2 and FOXO4 in above treated CAL27, SCC-9 and SCC-I5 cells. (E) Relative expression levels of PHLPP2 and FOXO4 in 68 pairs of HNSCC and NAT tissues. (F) Pearson correlation analysis between PHLPP2/FOXO4 mRNA and miR-762 expression in HNSCC. ${ }^{* *} p<0.01$.

participate in the promotion of miR-762 on the proliferation and migration of HNSCC cells. We transfected overexpression plasmid pc-PHLPP2 or pc-FOXO4 into miR-762overexpressing CAL27 cells to upregulate their expression (Figure 5A). CCK-8 and IncuCyte assays revealed that proliferation and migration were decreased after we ectopically restored PHLPP2 and FOXO4 expression (Figure 5B). Concordantly, the knockdown of PHLPP2 and FOXO4 enhanced the proliferation and migration of CAL27 cells (Figure 5C and D). Western blot analyses demonstrated that overexpression of PHLPP2 or FOXO4 reversed the effects of miR-762 on the expression of several proteins (Figure 5E); the expression of $\mathrm{p}$-AKT, N-cadherin, and vimentin was decreased, but E-cadherin expression was increased. By contrast, silencing of PHLPP2 and FOXO4 with siRNAs had similar effects to those observed with miR-762 upregulation, showing enhanced p-AKT, N-cadherin, and vimentin expression, and suppressed E-cadherin expression (Figure 5F). These results indicated that PHLPP2 and $\mathrm{FOXO} 4$ are functional downstream mediators of miR-762 in HNSCC.

\section{miR-762 Promotes Tumor Growth in vivo}

We established a xenograft mouse tumor model to explore the function of miR-762 on HNSCC cell growth in vivo. CAL27 cells were infected with the lentivirus particles Lenti-miR-762 or Lenti-Mock, and infection efficiency was confirmed via qRT-PCR (Figure 6A). Then, transfected cells were inoculated subcutaneously into female nude mice. Tumor volume was monitored every 4 days, and nude mice were sacrificed after 4 weeks of consecutive observation. The results showed a greater tumor volume and weight in the Lenti-miR-762 group than in the Lenti-Mock group of nude mice (Figure 6B-D). PHLPP2 and FOXO4 were distributed in cytoplasm and nucleus of cells and IHC analyses showed lower expression of PHLPP2 and FOXO4 in the Lenti-miR-762 group than in the Lenti-Mock group (Figure 6E). These observations indicated that miR-762 could effectively promote HNSCC growth in vivo.

\section{Discussion}

HNSCC shows aberrant expression of numerous miRNAs, which are involved in the initiation and progression of HNSCC. ${ }^{19-21}$ To our knowledge, this study is the first to reveal the role of miR-762 in HNSCC. We detected miR762 expression in HNSCC tissues and cell lines and investigated the clinical significance of miR-762 in patients with HNSCC. Furthermore, we assessed the biological effects of miR-762 on HNSCC progression in vitro and in vivo and explored the potential tumor-promoting mechanism of miR-762 in HNSCC cells. 

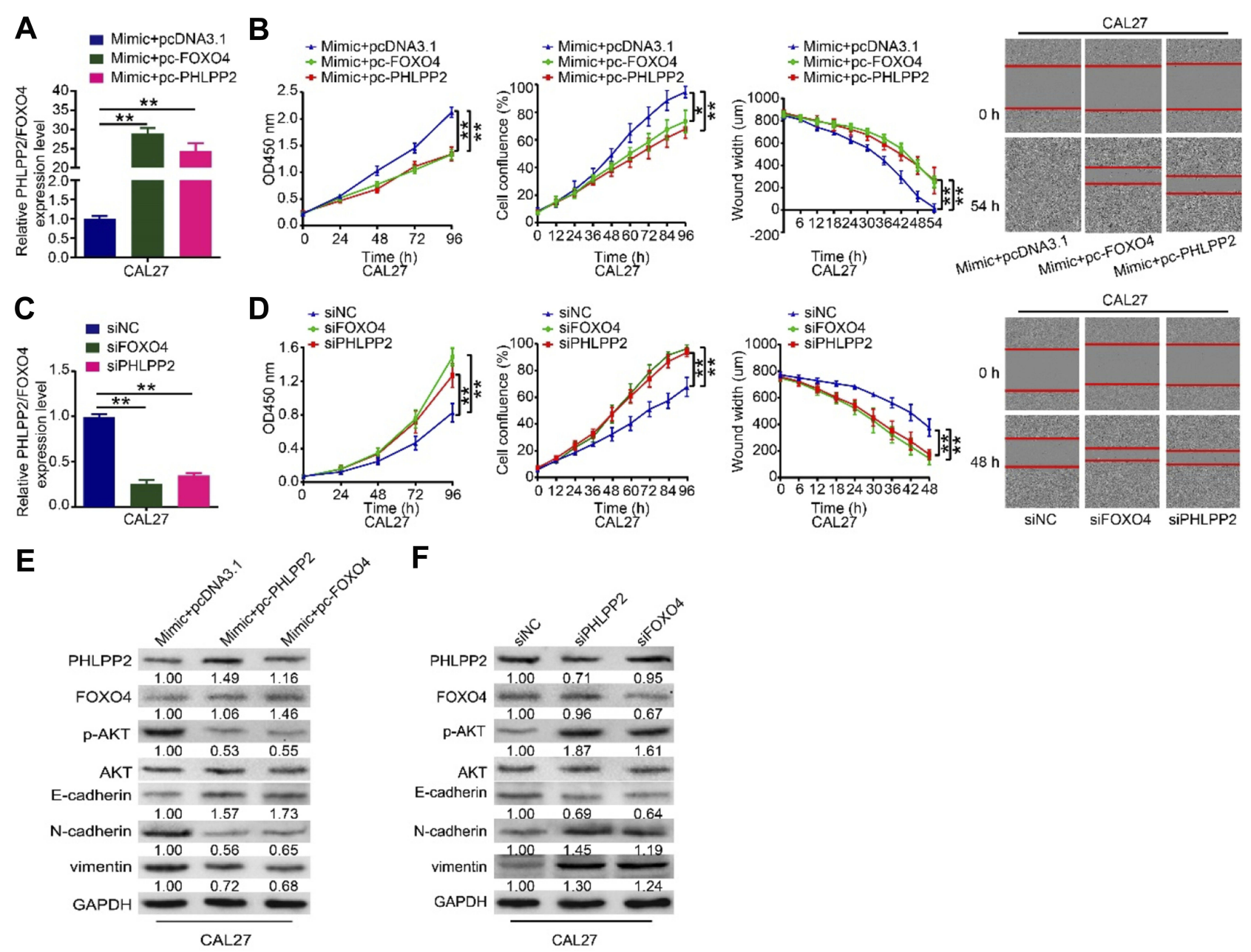

Figure 5 PHLPP2 and FOXO4 mediated the tumor-promotive effects of miR-762 on HNSCC cells.

Notes: (A) miR-762 mimics, along with pcDNA3.I, pc-PHLPP2 or pc-FOXO4, were co-transfected into CAL27 cells. Relative expression levels of PHLPP2 and FOXO4 of above treated CAL27 cells were detected by RT-qPCR; (B) The proliferation and migration ability of above treated CAL27 cells were measured using CCK-8 and IncuCyte assays; (C) Relative expression levels of PHLPP2 and FOXO4 in CAL27 cells transfected with siPHLPP2, siFOXO4 or siNC were detected by RT-qPCR. (D) The proliferation and migration ability of CAL27 cells transfected with siPHLPP2, siFOXO4 or siNC were measured by CCK-8 and IncuCyte assays; (E) Western blot analysis of PHLPP2, FOXO4, P-AKT, E-cadherin, N-cadherin and vimentin in above treated CAL27 cells. (F) Western blot analysis of PHLPP2, FOXO4, P-AKT, E-cadherin, N-cadherin and vimentin of CAL27 cells transfected with siPHLPP2, siFOXO4 or siNC. *p $<0.05, * * p<0.01$.

Previous studies revealed upregulated miR-762 expression in breast cancer cell lines and tissues. ${ }^{16}$ Similarly, miR-762 is abnormally elevated in ovarian cancer tissues and cell lines, and patients with high levels of miR-762 expression show significantly worse overall survival than those with low levels of expression. ${ }^{17}$ Notably, miR-762 is significantly upregulated in animal model of oral squamous cell carcinoma in an Syrian hamster. ${ }^{22}$

Herein, we showed upregulated miR-762 expression in HNSCC tissues and cell lines. The high miR-762 expression was significantly correlated with TNM stage of HNSCC patients. Furthermore, HNSCC patients with high-expressing miR-762 had a shorter overall survival time than those with low-expressing miR-762. These findings suggest that miR762 might be a diagnostic or prognostic molecule of HNSCC.
miR-762 has been identified as a tumor promoter in breast cancer, overexpressed miR-762 facilitates the proliferation and invasion of breast cancer cells. ${ }^{16}$ In addition, enhanced miR-762 expression facilitates the proliferation and apoptosis resistance through the Wnt/ß-catenin signaling pathway in ovarian cancer. ${ }^{17}$ Furthermore, increased miR-762 expression promotes the migration and invasion of ovarian cancer and muscle-invasive bladder cancer cells. ${ }^{17,23}$ However, the specific roles of miR-762 in HNSCC remain unclear. Here, we showed that miR-762 had tumor-promotive effects on the malignant characteristics of HNSCC, promoting cancer cell proliferation and migration in vitro and tumor growth in vivo. In addition, pathway enrichment analyses uncovered the close relationship between miR-762 and the PI3K pathway. Western 


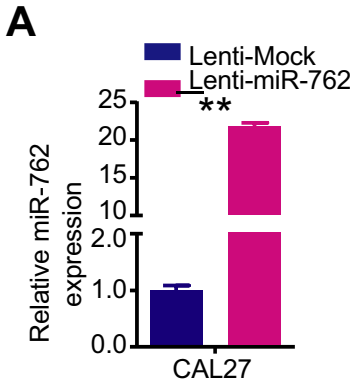

E
B

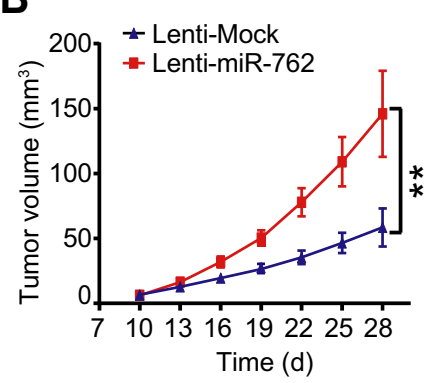

C

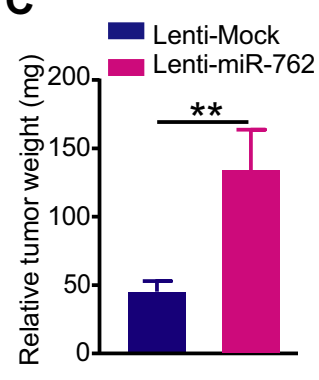

D

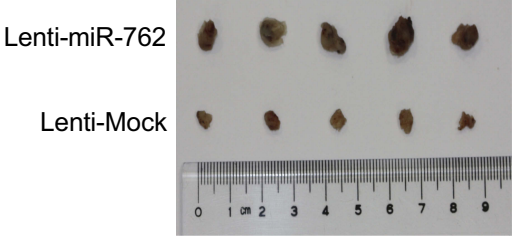

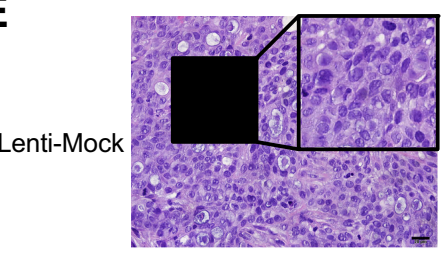
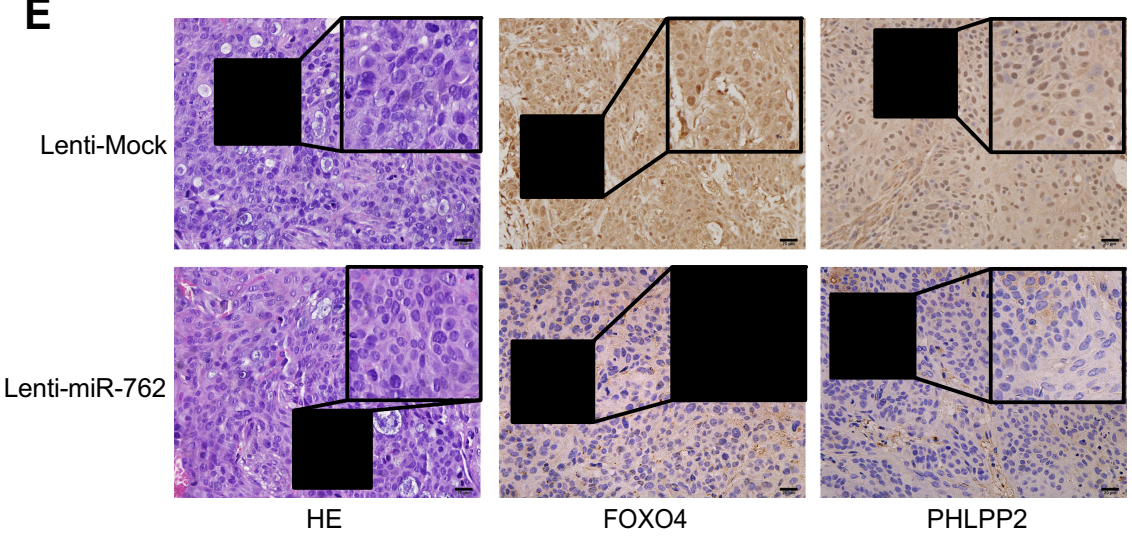

PHLPP2

Figure 6 miR-762 accelerated tumor growth of HNSCC cells in vivo.

Notes: (A) CAL27 cells transfected with Lenti-miR-762 or Lenti-Mock, and then were subcutaneously injected into female nude mice. The expression of miR-762 was examined by RT-qPCR in two groups of nude mice. (B and C) The growth curve and weights of tumors of two groups of nude mice. (D) Photographs of tumors derived from two groups of nude mice. (E) The xenograft tumor tissues of nude mice were assessed by H\&E staining in two groups ( $\times 400$ ); The expression levels of PHLPP2 and FOXO4 in xenografted tumor were tested by immunohistochemistry in two groups. The regions in boxes were enlarged and shown as inserts. Magnification $\times 400 ;$ scale bar represents 20 um. ${ }^{* *} p<0.01$.

blot analyses revealed that miR-762 could activate the AKT signaling pathway and promote the EMT in HNSCC cells. These results suggest that miR-762 could be a promising therapeutic target in HNSCC.

miRNAs function mainly by directly regulating the mRNA of numerous downstream genes. Multiple targets of miR-762 have been identified, including RNase7, ST2, IRF7 and menin. ${ }^{16,17,24}$ Here, PHLPP2 and FOXO4 mRNAs were screened as putative targets of miR-762 via bioinformatics algorithms and microarrays. PHLPP2 has been identified as a tumor suppressor in hepatocellular carcinoma, ovarian cancer, colorectal cancer, and gastric cancer, and downregulation of PHLPP2 promotes proliferation and invasion of these cancers. $^{25-28}$ FOXO4, a member of the FOXO family, plays an important role in tumor suppression. Overexpression of FOXO4 induces apoptosis in clear-cell renal carcinoma cells. $^{29}$ Forced expression of FOXO4 prevents the growth and metastasis of gastric cancer in vitro and dramatically restricts tumor growth in vivo. ${ }^{30}$

In the present study, luciferase activity reporter assays, qRT-PCR, and Western blot analyses confirmed that PHLPP2 and FOXO4 were targets of miR-762. Importantly, the mRNA levels of PHLPP2 and FOXO4 were inversely correlated with miR-762 expression level in HNSCC tissues. Rescue assays were performed to investigate whether the effects of miR-762 in regulating HNSCC could be attributed to its regulation of PHLPP2 and FOXO4. The results showed that knockdown of PHLPP2 and FOXO4 mimicked the tumor-promotive effects of miR-762 in HNSCC. By contrast, overexpression of PHLPP2 or FOXO4 could abrogate the promotive effects of miR-762 on proliferation and migration of HNSCC cells. As a phosphatase, PHLPP2 dephosphorylates AKT at serine 473 to reduce AKT activity. ${ }^{31}$ Suppression of PHLPP2 leads to upregulation of p-AKT and acceleration of the EMT in gastric cancer. ${ }^{32}$ Downregulated FOXO4 not only promotes proliferation and metastasis of cervical cancer cells, but also facilitates the EMT. ${ }^{33}$ Western blot assays demonstrated that miR-762 mimic and knockdown of PHLPP2 and FOXO4 enhanced p-AKT expression, and facilitated the EMT in HNSCC cells. However, miR-762 inhibitor and overexpression of PHLPP2 or FOXO4 could reverse the influence of miR-762 on the above proteins. These data suggested that miR-762 promotes cell proliferation and migration by modulating PHLPP2 and FOXO4. 


\section{Conclusion}

HNSCC samples showed upregulated expression of miR762 , which promoted cell proliferation and migration. miR-762 directly targeted PHLPP2 and FOXO4 to regulate p-AKT expression and the EMT in HNSCC cells. These results suggest that miR-762 might be a potential therapeutic target for HNSCC.

\section{Acknowledgments}

This work was supported by research grants from the National Nature Science Foundation of China (81671006, 81030018 and 81300894), the Beijing Nature Science Foundation (7172238) and Chinese Academy of Medical Sciences Research Unit, Peking University School and Hospital of Stomatology (2019RU034).

\section{Disclosure}

The authors report no conflicts of interest in this work.

\section{References}

1. Tumban E. A current update on human papillomavirus-associated head and neck cancers. Viruses. 2019;11(10):922. doi:10.3390/ v11100922

2. Siegel RL, Miller KD, Jemal A. Cancer statistics, 2019. CA Cancer J Clin. 2019;69(1):7-34. doi:10.3322/caac.21551

3. Chen W, Zheng R, Baade PD, et al. Cancer statistics in China, 2015. CA Cancer J Clin. 2016;66(2):115-132. doi:10.3322/caac.21338

4. Wu ZH, Tang Y, Niu X, Cheng Q. Expression and gene regulation network of INHBA in head and neck squamous cell carcinoma based on data mining. Sci Rep. 2019;9(1):14341. doi:10.1038/s41598-01950865-y

5. Li S, Lee YC, Li Q, et al. Oral lesions, chronic diseases and the risk of head and neck cancer. Oral Oncol. 2015;51(12):1082-1087. doi:10.1016/j.oraloncology.2015.10.014

6. Wang S, Jin S, Liu MD, et al. Hsa-let-7e-5p inhibits the proliferation and metastasis of head and neck squamous cell carcinoma cells by targeting chemokine receptor 7. J Cancer. 2019;10(8):1941-1948. doi: $10.7150 /$ jca. 29536

7. Yu SS, Cirillo N. The molecular markers of cancer stem cells in head and neck tumors. J Cell Physiol. 2019. doi:10.1002/jcp.28963

8. Jiang L, Zhao Z, Zheng L, Xue L, Zhan Q, Song Y. Downregulation of miR-503 promotes ESCC cell proliferation, migration, and invasion by targeting cyclin D1. Genomics Proteomics Bioinformatics. 2017;15(3):208-217. doi:10.1016/j.gpb.2017.04.003

9. Liu R, Guo H, Lu S. MiR-335-5p restores cisplatin sensitivity in ovarian cancer cells through targeting BCL2L2. Cancer Med. 2018 doi:10.1002/cam4.1682

10. Nguyen TH, Liu X, Su ZZ, Hsu AC, Foster PS, Yang M. Potential role of MicroRNAs in the regulation of antiviral responses to influenza infection. Front Immunol. 2018;9:1541. doi:10.3389/fimmu.2018.01541

11. Zhao M, Luo R, Liu Y, et al. miR-3188 regulates nasopharyngeal carcinoma proliferation and chemosensitivity through a FOXO1-modulated positive feedback loop with mTOR-p-PI3K/AKT-c-JUN. Nat Commun. 2016;7:11309. doi:10.1038/ncomms11309

12. Kleemann M, Schneider H, Unger K, et al. MiR-744-5p inducing cell death by directly targeting HNRNPC and NFIX in ovarian cancer cells. Sci Rep. 2018;8(1):9020. doi:10.1038/s41598-018-27438-6
13. Zhao Z, Fan $\mathrm{X}$, Jiang $\mathrm{L}$, et al. miR-503-3p promotes epithelial-mesenchymal transition in breast cancer by directly targeting SMAD2 and E-cadherin. J Genet Genom. 2017;44(2):75-84. doi:10.1016/j.jgg.2016.10.005

14. Zhao Z, Gao D, Ma T, Zhang L. MicroRNA-141 suppresses growth and metastatic potential of head and neck squamous cell carcinoma. Aging. 2019;11(3):921-932. doi:10.18632/aging.101791

15. Kim EJ, Kim JS, Lee S, et al. QKI, a miR-200 target gene, suppresses epithelial-to-mesenchymal transition and tumor growth. Int $j$ Cancer. 2019. doi:10.1002/ijc.32372

16. Li Y, Huang R, Wang L, et al. microRNA-762 promotes breast cancer cell proliferation and invasion by targeting IRF7 expression. Cell Prolif. 2015;48(6):643-649. doi:10.1111/cpr.12223

17. Hou R, Yang Z, Wang S, et al. miR-762 can negatively regulate menin in ovarian cancer. Onco Targets Ther. 2017;10:2127-2137. doi:10.2147/OTT.S127872

18. Chen X, Xie W, Gu P, et al. Upregulated WDR5 promotes proliferation, self-renewal and chemoresistance in bladder cancer via mediating H3K4 trimethylation. Sci Rep. 2015;5:8293. doi:10.1038/srep08293

19. Lu S, Zhou C, Zou B, Zhang H, Feng M. MiR-4295 facilitates cell proliferation and metastasis in head and neck squamous cell carcinoma by targeting NPTX1. Genes Immun. 2019. doi:10.1038/s41435019-0081-0

20. Yang CX, Sedhom W, Song J, Lu SL. The role of MicroRNAs in recurrence and metastasis of head and neck squamous cell carcinoma. Cancers. 2019;11(3). doi:10.3390/cancers11030395

21. Kolenda T, Guglas K, Teresiak A, Blizniak R, Lamperska K. Low let-7d and high miR-205 expression levels positively influence HNSCC patient outcome. J Biomed Sci. 2019;26(1):17. doi:10.1186/s12929-019-0511-3

22. Yu T, Wang XY, Gong RG, et al. The expression profile of microRNAs in a model of 7,12-dimethyl-benz[a]anthrance-induced oral carcinogenesis in Syrian hamster. $J$ Exp Clin Cancer Res. 2009;28:64. doi:10.1186/1756-9966-28-64

23. Lin G, Sheng H, Xie H, et al. circLPAR1 is a novel biomarker of prognosis for muscle-invasive bladder cancer with invasion and metastasis by miR-762. Oncol Lett. 2019;17(3):3537-3547. doi:10.3892/ ol.2019.9970

24. Mun J, Tam C, Chan G, Kim JH, Evans D, Fleiszig S. MicroRNA-762 is upregulated in human corneal epithelial cells in response to tear fluid and Pseudomonas aeruginosa antigens and negatively regulates the expression of host defense genes encoding RNase7 and ST2. PLoS One. 2013;8(2): e57850. doi:10.1371/journal.pone.0057850

25. Li CF, Li YC, Jin JP, Yan ZK, Li DD. miR-938 promotes colorectal cancer cell proliferation via targeting tumor suppressor PHLPP2. Eur J Pharmacol. 2017;807:168-173. doi:10.1016/j.ejphar.2017.04.023

26. Ding L, Zhang S, Xu M, Zhang R, Sui P, Yang Q. MicroRNA-27a contributes to the malignant behavior of gastric cancer cells by directly targeting PH domain and leucine-rich repeat protein phosphatase 2.J Exp Clin Cancer Res. 2017;36(1):45. doi:10.1186/s13046-017-0516-2

27. Liao Y, Deng Y, Liu J, et al. MiR-760 overexpression promotes proliferation in ovarian cancer by downregulation of PHLPP2 expression. Gynecol Oncol. 2016;143(3):655-663. doi:10.1016/j. ygyno.2016.09.010

28. Cui X, Li Q, He Y. miR-3117 regulates hepatocellular carcinoma cell proliferation by targeting PHLPPL. Mol Cell Biochem. 2017;424(1-2):195-201. doi:10.1007/s11010-016-2855-2

29. Wang W, Zhou PH, Hu W. Overexpression of FOXO4 induces apoptosis of clear-cell renal carcinoma cells through downregulation of Bim. Mol Med Rep. 2016;13(3):2229-2234. doi:10.3892/mmr.2016.4789

30. Su L, Liu X, Chai N, et al. The transcription factor FOXO4 is down-regulated and inhibits tumor proliferation and metastasis in gastric cancer. BMC Cancer. 2014;14:378. doi:10.1186/1471-2407-14-378

31. Brognard J, Sierecki E, Gao T, Newton AC. PHLPP and a second isoform, PHLPP2, differentially attenuate the amplitude of Akt signaling by regulating distinct Akt isoforms. Mol Cell. 2007;25(6):917-931. doi:10.1016/j.molcel.2007.02.017 
32. Jiang $\mathrm{L}$, Wang $\mathrm{C}$, Lei $\mathrm{F}$, et al. miR-93 promotes cell proliferation in gliomas through activation of PI3K/Akt signaling pathway. Oncotarget. 2015;6(10):8286-8299. doi:10.18632/oncotarget.3221
33. Li J, Hu L, Tian C, Lu F, Wu J, Liu L. microRNA-150 promotes cervical cancer cell growth and survival by targeting FOXO4. BMC Mol Biol. 2015;16:24. doi:10.1186/s12867-015-0052-6

\section{Publish your work in this journal}

OncoTargets and Therapy is an international, peer-reviewed, open access journal focusing on the pathological basis of all cancers, potential targets for therapy and treatment protocols employed to improve the management of cancer patients. The journal also focuses on the impact of management programs and new therapeutic

Submit your manuscript here: https://www.dovepress.com/oncotargets-and-therapy-journal agents and protocols on patient perspectives such as quality of life, adherence and satisfaction. The manuscript management system is completely online and includes a very quick and fair peer-review system, which is all easy to use. Visit http://www.dovepress.com/ testimonials.php to read real quotes from published authors. 